\title{
The Twisting Bifurcations of Double Homoclinic Loops with Resonant Eigenvalues
}

\author{
Xiaodong Li, ${ }^{1}$ Weipeng Zhang,, ${ }^{1}$ Fengjie Geng, ${ }^{2}$ and Jicai Huang ${ }^{3}$ \\ ${ }^{1}$ School of Mathematics and Statistics, Northeast Normal University, Changchun, Jilin 130024, China \\ ${ }^{2}$ School of Information Engineering, China University of Geosciences (Beijing), Beijing 100083, China \\ ${ }^{3}$ School of Mathematics and Statistics, Central China Normal University, Wuhan, Hubei 430079, China \\ Correspondence should be addressed to Weipeng Zhang; wpzhang808@163.com
}

Received 19 February 2013; Accepted 22 April 2013

Academic Editor: Maoan Han

Copyright (C) 2013 Xiaodong Li et al. This is an open access article distributed under the Creative Commons Attribution License, which permits unrestricted use, distribution, and reproduction in any medium, provided the original work is properly cited.

\begin{abstract}
The twisting bifurcations of double homoclinic loops with resonant eigenvalues are investigated in four-dimensional systems. The coexistence or noncoexistence of large 1-homoclinic orbit and large 1-periodic orbit near double homoclinic loops is given. The existence or nonexistence of saddle-node bifurcation surfaces is obtained. Finally, the complete bifurcation diagrams and bifurcation curves are also given under different cases. Moreover, the methods adopted in this paper can be extended to a higher dimensional system.
\end{abstract}

\section{Introduction and Setting of the Problem}

In recent years, there is a large literature concerning the bifurcation problems of homoclinic and heteroclinic loops in dynamical systems (see [1-23] and the references therein). However, to the best of the authors' knowledge, less attention has been devoted to the bifurcation of double homoclinic loops. Han and $\mathrm{Bi}$ [24] investigated the existence of homoclinic bifurcation curves and small and large limit cycles bifurcated from a double homoclinic loop under multiple parameter perturbations for general planar systems. Han and Chen [25] gave the number of limit cycles near double homoclinic loops under perturbations in planar Hamiltonian systems. Homburg and Knobloch [26] considered the existence of two homoclinic orbits in the bellows configuration, where the homoclinic orbits approach the equilibrium along the same direction for positive and negative times in conservative and reversible systems. Morales et al. [27, 28] presented contracting and expanding Lorenz attractors through resonant double homoclinic loops. Lu [29] obtained codimension 2 bifurcations of twisted double homoclinic loops in higher dimensional systems. Ragazzo [30] investigated the stability of sets that were generalizations of the simple pendulum double homoclinic loop. In our recent work [31, 32], codimension 2 bifurcations of double homoclinic loops and codimension 3 bifurcations of nontwisted double homoclinic loops with resonant eigenvalues were studied. Concerning this topic, a more extensive list of references can be found in the references mentioned earlier. Generally speaking, when studying the problem of single homoclinic or heteroclinic bifurcation connecting hyperbolic equilibrium, the bifurcation is more complicated as $\Gamma$ is twisted. Moreover, it is known that double homoclinic loops have higher codimension than a single homoclinic loop under the same conditions. Therefore, it will be more challenging and difficult to analyze the twisting bifurcations of double homoclinic loops.

In this paper, on the one hand, using the method which was originally established in $[22,23]$ and then improved in $[9,17,21]$, and so forth, we study the bifurcations of double homoclinic loops with resonant eigenvalues under twisted cases. Besides, the method is more applicable and bifurcation equations obtained in this paper are easier to compute. On the other hand, frequently, the too many equivalent terms in the bifurcation equation will make the bifurcation equation more complex so that it is very difficult 
to analyze the bifurcation equation. Applying the method used by Homburg and Knobloch [26] to analyze the centerstable and center-unstable tangent bundles, we cannot only get a smooth coordinate transformation in the neighborhood of $\Gamma$ small enough, but also make the bifurcation equation definite under an additional condition. Such strategy in dealing with the problems of bifurcations from homoclinic and heteroclinic loops is rarely used in the existing literatures. Therefore, a main feature in this paper is a combination of geometrical and analytical methods.

Motivated by these points, we will consider the following $C^{r}$ system and its unperturbed system:

$$
\begin{aligned}
& \dot{z}=f(z)+g(z, \mu), \\
& \dot{z}=f(z),
\end{aligned}
$$

where $r$ is large enough, $z \in R^{4}, \mu \in R^{l}, l \geq 3,0<|\mu| \ll$ $1, f(0)=0, g(0, \mu)=g(z, 0)=0$.

We make the following assumptions, which are shown in Figure 1.

$\left(H_{1}\right)$ The linearization $D f(0)$ has simple real eigenvalues at the equilibrium $0:-\rho_{2},-\rho_{1}, \lambda_{1}, \lambda_{2}$ satisfying

$$
-\rho_{2}<-\rho_{1}<0<\lambda_{1}<\lambda_{2}, \quad \rho_{1}=\lambda_{1} .
$$

$\left(H_{2}\right)$ System (2) has double homoclinic loops $\Gamma=\Gamma_{1} \cup$ $\Gamma_{2}, \Gamma_{i}=\left\{z=r_{i}(t): t \in R, r_{i}( \pm \infty)=0\right\}$ and $\operatorname{dim}\left(T_{r_{i}(t)} W^{\mathcal{s}} \cap T_{r_{i}(t)} W^{\mathcal{u}}\right)=1, i=1,2$, where $W^{s}$ and $W^{u}$ are the stable and unstable manifolds of 0 , respectively.

$\left(H_{3}\right)$ Let $e_{i}^{ \pm}=\lim _{t \rightarrow \mp \infty}\left(\dot{r}_{i}(t) /\left|\dot{r}_{i}(t)\right|\right)$, and $e_{i}^{+} \in T_{0} W^{u}, e_{i}^{-} \in$ $T_{0} W^{s}$ unit eigenvectors corresponding to $\lambda_{1}$ and $-\rho_{1}$, respectively, and satisfying $e_{1}^{+}=-e_{2}^{+}, e_{1}^{-}=-e_{2}^{-}$.

$\left(H_{4}\right) \operatorname{Span}\left\{T_{r_{i}(t)} W^{u}, T_{r_{i}(t)} W^{s}, e_{i}^{+}\right\}=R^{4}$ as $t \gg 1$, and $\operatorname{Span}\left\{T_{r_{i}(t)} W^{u}, T_{r_{i}(t)} W^{s}, e_{i}^{-}\right\}=R^{4}$ as $t \ll-1, i=1,2$.

As shown in Figure 1, under the hypotheses $\left(H_{1}\right)-\left(H_{4}\right)$, we can see that the double homoclinic loops $\Gamma$ are of codimension 3 .

The single homoclinic loop in high-dimensional systems has been investigated by many authors (see $[1,2,4-8,13-$ $15,17,21,22]$ and the references therein). In this paper, we only focus on bifurcations of the large loop; that is, the double loops $\Gamma=\Gamma_{1} \cup \Gamma_{2}$.

A nondegenerate homoclinic orbit is called a nontwisted homoclinic orbit if the unstable manifold $W^{u}$ has an even number of half-twists along the homoclinic orbit, and is called a twisted homoclinic orbit if $W^{u}$ has an odd number of half-twists along the homoclinic orbit; see more details in Deng [4]. A characterization for a twisted homoclinic orbit is that it arises from and tends to the equilibrium point from different sides of the unstable manifold. However, a nontwisted homoclinic orbit does so from the same side of the manifold. For the double homoclinic loop, $\Gamma=\Gamma_{1} \cup \Gamma_{2}$, it is called double twisted if both $\Gamma_{1}$ and $\Gamma_{2}$ are twisted, single twisted if and only if one of them is twisted, and nontwisted otherwise.

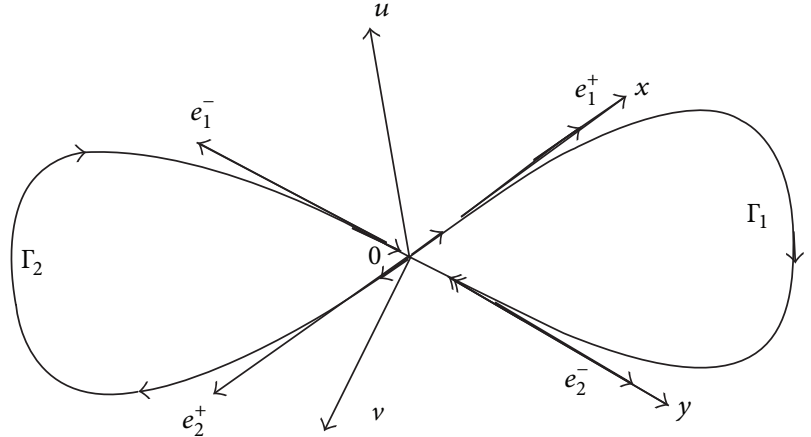

Figure 1: Double homoclinic loops $\Gamma=\Gamma_{1} \cup \Gamma_{2}$.

The rest of this paper is organized as follows. In Section 2, the normal form in a neighborhood of the equilibrium small enough is established and the bifurcation equations are given. In Section 3, by bifurcation analysis, the results of twisting bifurcations and complete bifurcation diagrams are obtained under different cases. We end the paper with a conclusion in Section 4.

\section{Normal Form and Bifurcation Equations}

From the hypothesis $\left(H_{1}\right)$, we can see that we confine ourselves to consider the resonance taking place between the two principal eigenvalues $\lambda_{1}$ and $-\rho_{1}$. Moreover, the corresponding eigenvectors are also the tangent directions of the homoclinic orbits. For simplicity, we may choose a new parameter $\mu=(\alpha, \nu)$ such that $\rho_{1}(\mu)=\lambda_{1}(\nu)+\alpha \lambda_{1}(\nu)$, $-1 \ll \alpha \ll 1$.

Let $U$ be a neighborhood of 0 small enough. By analyzing the center-stable and center-unstable tangent bundles, Homburg and Knobloch [26] obtained that there is a smooth coordinate transformation, such that in $U$ system (1) takes the following form:

$$
\begin{gathered}
\dot{x}=x\left(\lambda_{1}(v)+o(1)\right)+O(u)(O(y)+O(v)), \\
\dot{y}=y\left(-(1+\alpha) \lambda_{1}(v)+o(1)\right)+O(v)(O(x)+O(u)), \\
\dot{u}=u\left(\lambda_{2}(v)+o(1)\right)+x^{2} H_{1}(x, y, v), \\
\dot{v}=v\left(-\rho_{2}(v)+o(1)\right)+y^{2} H_{2}(x, y, u) .
\end{gathered}
$$

For the definiteness of the bifurcation equation, we make an additional assumption as follows:

$$
\begin{aligned}
& \left(H_{5}\right) H_{1}(x, 0,0)=0, H_{2}(0, y, 0)=0 \text {; that is, } \\
& H_{1}(x, y, v)=a_{1} x^{k_{1}} y^{k_{2}}+a_{2} x^{k_{3}} v^{k_{4}}+a_{3} x^{k_{5}} y^{k_{6}} v^{k_{7}}+\text { h.o.t., } \\
& H_{2}(x, y, u)=b_{1} y^{l_{1}} x^{l_{2}}+b_{2} y^{l_{3}} u^{l_{4}}+b_{3} x^{l_{5}} y^{l_{6}} u^{l_{7}}+\text { h.o.t., }
\end{aligned}
$$

where $k_{i}>\lambda_{2} / \lambda_{1}, i=1,3,5, k_{2} \rho_{1} / \lambda_{1}>2, k_{4} \rho_{2} / \lambda_{1}>2$, $\left(k_{6} \rho_{1}+k_{7} \rho_{2}\right) / \lambda_{1}>2 . l_{i}>\rho_{2} / \rho_{1}, i=1,3,5, l_{2} \lambda_{1} / \rho_{1}>2$, $l_{4} \lambda_{2} / \rho_{1}>2,\left(l_{6} \lambda_{1}+l_{7} \lambda_{2}\right) / \rho_{1}>2$. 
By the same process as in [32], which is based on the analysis of the Poincaré return map defined on some local transversal section of the double homoclinic loop $\Gamma$, we obtain the bifurcation equations as follows:

$$
\begin{aligned}
& s_{2}=\left(w_{1}^{12}\right)^{-1} s_{1}^{1+\alpha}-\delta^{-1} M_{1}^{1} v+\text { h.o.t., } \\
& s_{1}=\left(w_{2}^{12}\right)^{-1} s_{2}^{1+\alpha}+\delta^{-1} M_{2}^{1} v+\text { h.o.t. }
\end{aligned}
$$

\section{Bifurcation Analysis}

Denote that $w_{i}^{12}=\Delta_{i}\left|w_{i}^{12}\right|$. We say that $\Gamma$ is nontwisted as $\Delta_{1}=\Delta_{2}=1$ and twisted as $\Delta_{1}=\Delta_{2}=-1$ or $\Delta_{1} \Delta_{2}=$ -1 . In this paper, we focus on the twisted bifurcations. It is easy to see that $\left|w_{i}^{12}\right|$ is the approximate expanding rate of the solution $z_{i}^{1}(t)$ from $T_{i}^{0}$ to $-T_{i}^{1}$.

Case $1\left(\Delta_{1}=\Delta_{2}=-1\right.$ (i.e., double twisted)). For convenience and simplicity, we use the following notations throughout Case 1:

$$
\begin{aligned}
R_{1}^{1}= & \left\{v: M_{1}^{1} v>0, M_{2}^{1} v<0,|v| \ll 1\right\}, \\
R_{2}^{1}= & \left\{v: M_{1}^{1} v<0, M_{2}^{1} v>0,\left|M_{1}^{1} v\right|=O\left|M_{2}^{1} v\right|,|v| \ll 1\right\}, \\
D_{1}= & \left\{v \in R_{2}^{1}:\left(\delta^{-1} M_{1}^{1} v\right)^{1+\alpha}-w_{2}^{12}\left(2^{1 /(1+\alpha)}-1\right)\right. \\
& \left.\times \delta^{-1} M_{2}^{1} v>0 \text { for } \alpha>0\right\} \\
D_{2}= & \left\{v \in R_{2}^{1}:\left(-\delta^{-1} M_{2}^{1} v\right)^{1+\alpha}+w_{1}^{12}\left(2^{1 /(1+\alpha)}-1\right)\right. \\
& \left.\times \delta^{-1} M_{1}^{1} v>0 \text { for } \alpha>0\right\} .
\end{aligned}
$$

If (6) has solution $s_{1}=s_{2}=0$, then we have

$$
M_{i}^{1} \nu+\text { h.o.t. }=0, \quad i=1,2 .
$$

If $M_{i}^{1} \neq 0$, then there exists a codimension 1 surface $\Sigma_{i}$ with a normal vector $M_{i}^{1}$ at $\nu=0$, such that the $i$ th equation of (6) has solution $s_{1}=s_{2}=0$ as $\nu \in \Sigma_{i}$ and $|\nu| \ll 1$; that is, $\Gamma_{i}$ is persistent. If $\operatorname{rank}\left(M_{1}^{1}, M_{2}^{1}\right)=2$, then $\Sigma_{12}=\Sigma_{1} \cap \Sigma_{2}$ is a codimension 2 surface with a normal plane $\operatorname{span}\left\{M_{1}^{1}, M_{2}^{1}\right\}$ such that (6) has solution $s_{1}=s_{2}=0$ as $v \in \Sigma_{12}$ and $|\nu| \ll 1$; equivalently, the large loop $\Gamma=\Gamma_{1} \cup \Gamma_{2}$ is persistent.

Suppose that (6) has solution $s_{1}=0, s_{2}>0$. Then, we have

$$
\begin{gathered}
s_{2}=-\delta^{-1} M_{1}^{1} \nu+\text { h.o.t., } \\
0=\left(w_{2}^{12}\right)^{-1} s_{2}^{1+\alpha}+\delta^{-1} M_{2}^{1} \nu+\text { h.o.t. }
\end{gathered}
$$

Hence, we obtain the large 1-homoclinic orbit bifurcation surface equation

$$
H_{2}^{1}: \delta^{-1} w_{2}^{12} M_{2}^{1} \nu+\left(-\delta^{-1} M_{1}^{1} \nu\right)^{1+\alpha}+\text { h.o.t. }=0,
$$

which is well defined at least in the region $R_{2}^{1}$ and has a normal vector $M_{2}^{1}$ as $\alpha>0$ (resp., $M_{1}^{1}$ as $\alpha<0$ ) at $\nu=0$ such that system (1) has a large 1-homoclinic loop $\Gamma_{2}^{1}$ near $\Gamma$ as $v \in H_{2}^{1}$. It also means that $H_{2}^{1}$ is tangent to $\Sigma_{2}$ as $\alpha>0$ (resp., $\Sigma_{1}$ as $\alpha<$ $0)$. When $v \in H_{2}^{1}, \alpha>0, s_{1}=0$, and $s_{2}=-\delta^{-1} M_{1}^{1} \nu+$ h.o.t., (6) indicates that

$$
\begin{gathered}
s_{2 v}=-\delta^{-1} M_{1}^{1}+\text { h.o.t. } \\
s_{1 v}=(1+\alpha)\left(w_{2}^{12}\right)^{-1}\left(-\delta^{-1} M_{1}^{1} v\right)^{\alpha} s_{2 v}+\delta^{-1} M_{2}^{1}+\text { h.o.t. }
\end{gathered}
$$

Using (11) $\times(1+\alpha)\left(w_{2}^{12}\right)^{-1}\left(-\delta^{-1} M_{1}^{1} v\right)^{\alpha}+(12)$, we have

$$
\left.s_{1 \nu}\right|_{H_{2}^{1}}=\delta^{-1} M_{2}^{1}+O\left(\left|M_{1}^{1} \nu\right|^{\alpha}\right) \text {. }
$$

So, $s_{1}=s_{1}(\nu)$ increases along the direction of the gradient $M_{2}^{1}$ for $\nu$ near $H_{2}^{1}$.

Similarly, by setting $s_{k}=e^{-\rho_{1} \tau_{k}}$, we can derive that $s_{1}(\nu)$ increases along the direction $-M_{1}^{1}$ for $v$ near $H_{2}^{1}$ as $\alpha<0$.

If (6) has solution $s_{1}>0$ and $s_{2}=0$, then we have

$$
\begin{gathered}
0=\left(w_{1}^{12}\right)^{-1} s_{1}^{1+\alpha}-\delta^{-1} M_{1}^{1} v+\text { h.o.t. } \\
s_{1}=\delta^{-1} M_{2}^{1} v+\text { h.o.t. }
\end{gathered}
$$

Therefore, we obtain the existence of the other large 1homoclinic orbit bifurcation surface equation

$$
H_{1}^{1}: \delta^{-1} w_{1}^{12} M_{1}^{1} v-\left(\delta^{-1} M_{2}^{1} v\right)^{1+\alpha}+\text { h.o.t. }=0 \text {, }
$$

which is well defined at least in the region $R_{2}^{1}$ and has a normal vector $M_{1}^{1}$ as $\alpha>0$ (resp., $M_{2}^{1}$ as $\alpha<0$ ) at $\nu=0$ such that system (1) has a large 1-homoclinic loop $\Gamma_{1}^{1}$ near $\Gamma$ as $v \in H_{1}^{1}$. Thus, $H_{1}^{1}$ is also tangent to $\Sigma_{1}$ as $\alpha>0$ (resp., $\Sigma_{2}$ as $\alpha<0$ ). For $v \in H_{1}^{1}$ and $\alpha>0, s_{1}=\delta^{-1} M_{2}^{1} v+$ h.o.t., $s_{2}=0$, it follows from (6) that

$$
\begin{gathered}
s_{2 v}=(1+\alpha)\left(w_{1}^{12}\right)^{-1}\left(\delta^{-1} M_{2}^{1} v\right)^{\alpha} s_{1 v}-\delta^{-1} M_{1}^{1}+\text { h.o.t. } \\
s_{1 v}=\delta^{-1} M_{2}^{1}+\text { h.o.t. }
\end{gathered}
$$

Computing $(17) \times(1+\alpha)\left(w_{1}^{12}\right)^{-1}\left(\delta^{-1} M_{2}^{1} \nu\right)^{\alpha}+(16)$, it leads to

$$
\left.s_{2 \nu}\right|_{H_{1}^{1}}=-\delta^{-1} M_{1}^{1}+O\left(\left|M_{2}^{1} \nu\right|^{\alpha}\right) .
$$

So, $s_{2}=s_{2}(\nu)$ increases along the direction $-M_{1}^{1}$ for $v$ close to $H_{1}^{1}$.

By a similar procedure, we can show that $s_{2}(\nu)$ increases along the direction $M_{2}^{1}$ as $v$ is in the neighborhood of $H_{1}^{1}$ and $\alpha<0$.

Summing up the previous analysis, we have the following theorem.

Theorem 1. Suppose that $\left(H_{1}\right)-\left(H_{5}\right)$ hold; then, the following conclusions are true.

(1) If $M_{i}^{1} \neq 0$, then there exists a unique surface $\Sigma_{i}$ with codimension 1 and normal vectors $M_{i}^{1}$ at $v=0$, such that system (1) has a homoclinic loop near $\Gamma_{i}$ if and only 
if $\nu \in \Sigma_{i}$ and $|\nu| \ll 1$. If $\operatorname{rank}\left(M_{1}^{1}, M_{2}^{1}\right)=2$, then $\Sigma_{12}=\Sigma_{1} \cap \Sigma_{2}$ is a codimension 2 surface and $0 \in \Sigma_{12}$ such that system (1) has a large loop consisting of two homoclinic orbits near $\Gamma$ as $\nu \in \Sigma_{12}$ and $|\nu| \ll 1$; that is, $\Gamma$ is persistent.

(2) In the region $R_{2}^{1}$, there exists a large 1-homoclinic orbit bifurcation surface $H_{2}^{1}$ which is tangent to $\Sigma_{2}$ (resp., $\Sigma_{1}$ ) at $v=0$ with the normal vector $M_{2}^{1}$ (resp., $M_{1}^{1}$ ) as $\alpha>0$ (resp., $\alpha<0$ ), and for $\nu \in H_{2}^{1}$, system (1) has a unique large 1-homoclinic orbit near $\Gamma$. Furthermore, the unique large 1-homoclinic orbit near $\Gamma$ becomes a large 1-periodic orbit when $\nu$ moves along the direction $M_{2}^{1}$ (resp., $-M_{1}^{1}$ ) and nearby $H_{2}^{1}$ as $\alpha>0$ (resp., $\alpha<$ $0)$. Meanwhile, there exists another large 1-homoclinic orbit bifurcation surface $H_{1}^{1}$ which is tangent to $\Sigma_{1}$ (resp., $\Sigma_{2}$ ) at $v=0$ with the normal $M_{1}^{1}$ (resp., $M_{2}^{1}$ ) as $\alpha>0$ (resp., $\alpha<0$ ), and for $\nu \in H_{1}^{1}$, system (1) has another one unique large 1-homoclinic orbit near $\Gamma$. Furthermore, the unique large 1-homoclinic orbit near $\Gamma$ changes into a large 1-periodic orbit when $v$ shifts along the direction $-M_{1}^{1}$ (resp., $M_{2}^{1}$ ) and near $H_{1}^{1}$ as $\alpha>0$ (resp., $\alpha<0$ ).

Lemma 2. Suppose that hypotheses $\left(H_{1}\right)-\left(H_{5}\right)$ and $0<\alpha \ll 1$ are valid; then, in addition to the large 1-homoclinic loop $\Gamma_{2}^{1}$, system (1) has exactly one simple large 1-periodic orbit near $\Gamma$ for $v \in H_{2}^{1} \cap D_{2}$. Moreover, the large 1-periodic orbit is persistent as $v$ changes in the neighborhood of $\mathrm{H}_{2}^{1}$.

Proof. If $v \in H_{2}^{1}$ and $|\nu| \ll 1$, then we know that system (1) has one large 1-homoclinic loop $\Gamma_{2}^{1}$. Now, following (6), we have

$$
\begin{aligned}
& {\left[\left(w_{1}^{12}\right)^{-1} s_{1}^{1+\alpha}-\delta^{-1} M_{1}^{1} v+\text { h.o.t. }\right]^{1+\alpha}=w_{2}^{12} s_{1}-\delta^{-1} w_{2}^{12} M_{2}^{1} \nu} \\
& + \text { h.o.t. }
\end{aligned}
$$

Let $N_{1}\left(s_{1}\right)$ and $L_{1}\left(s_{1}\right)$ be the left- and right-hand sides of (19), respectively. Then, by (10), we get $N_{1}(0)=L_{1}(0)$ as $v \in H_{2}^{1}$. Moreover,

$$
\begin{gathered}
N_{1}^{\prime}\left(s_{1}\right)=(1+\alpha)^{2}\left(w_{1}^{12}\right)^{-1}\left[\left(w_{1}^{12}\right)^{-1} s_{1}^{1+\alpha}-\delta^{-1} M_{1}^{1} \nu+\text { h.o.t. }\right]^{\alpha} s_{1}^{\alpha}, \\
L_{1}^{\prime}\left(s_{1}\right)=w_{2}^{12}+\text { h.o.t. }
\end{gathered}
$$

Thus, $0=\left.N_{1}^{\prime}\left(s_{1}\right)\right|_{s_{1}=0}>\left.L_{1}^{\prime}\left(s_{1}\right)\right|_{s_{1}=0}$. Therefore, there is an $\widetilde{s}_{1}$, $0<\widetilde{s}_{1} \ll 1$, such that for $0<s_{1}<\widetilde{s}_{1}, N_{1}\left(s_{1}\right)>L_{1}\left(s_{1}\right)$.

Let $\bar{s}_{1}=-\delta^{-1} M_{2}^{1} \nu$. Then,

$$
\begin{gathered}
N_{1}\left(\bar{s}_{1}\right)=\left[\left(w_{1}^{12}\right)^{-1}\left(-\delta^{-1} M_{2}^{1} \nu\right)^{1+\alpha}-\delta^{-1} M_{1}^{1} \nu+\text { h.o.t. }\right]^{1+\alpha} \\
L_{1}\left(\bar{s}_{1}\right)=w_{2}^{12}\left(-\delta^{-1} M_{2}^{1} \nu\right)-\delta^{-1} w_{2}^{12} M_{2}^{1} v+\text { h.o.t. } \\
=2\left(-\delta^{-1} M_{1}^{1} v\right)^{1+\alpha}+\text { h.o.t. }
\end{gathered}
$$

which means that $N_{1}\left(\bar{s}_{1}\right)<L_{1}\left(\bar{s}_{1}\right)$ as $v \in H_{2}^{1} \cap D_{2}$. Then, it follows from $N_{1}^{\prime \prime}\left(s_{1}\right)<0$ that $N_{1}\left(s_{1}\right)=L_{1}\left(s_{1}\right)$ has a unique solution $s_{1}^{*}$ satisfying $0<\widetilde{s}_{1}<s_{1}^{*} \ll 1$, which is shown in Figure 2. That is, system (1) has a unique large 1-periodic loop near $\Gamma$ for $\nu \in H_{2}^{1} \cap D_{2}$ and $0<|\nu| \ll 1$. The proof is complete.

Lemma 3. Suppose that hypotheses $\left(H_{1}\right)-\left(H_{5}\right)$ and $0<\alpha \ll 1$ hold. Then, system (1) has a saddle-node bifurcation surface of large 1-periodic orbit in the region $R_{2}^{1} \cap\left\{\nu:\left|M_{1}^{1} \nu\right|=o\left(\left|M_{2}^{1} \nu\right|\right)\right\}$ as follows:

$$
\begin{aligned}
S N^{2} & :-\delta^{-1} w_{2}^{12} M_{2}^{1} \nu \\
= & {\left[\left(w_{1}^{12}\right)^{-1}\left(\frac{w_{1}^{12} M_{1}^{1} \nu}{(1+\alpha)^{2} M_{2}^{1} \nu}\right)^{(1+\alpha) / \alpha}-\delta^{-1} M_{1}^{1} \nu\right]^{1+\alpha} } \\
& -w_{2}^{12}\left(\frac{w_{1}^{12} M_{1}^{1} \nu}{(1+\alpha)^{2} M_{2}^{1} \nu}\right)^{1 / \alpha}+\text { h.o.t. }
\end{aligned}
$$

Proof. It is easy to see that $h=N_{1}\left(s_{1}\right)$ is tangent to $h=L_{1}\left(s_{1}\right)$ at some point $s_{1}$, as shown in Figure 3; if

$$
\begin{aligned}
& N_{1}\left(s_{1}\right)=L_{1}\left(s_{1}\right), \\
& N_{1}^{\prime}\left(s_{1}\right)=L_{1}^{\prime}\left(s_{1}\right),
\end{aligned}
$$

then

$$
\begin{aligned}
& {\left[\left(w_{1}^{12}\right)^{-1} s_{1}^{1+\alpha}-\delta^{-1} M_{1}^{1} v+\text { h.o.t. }\right]^{1+\alpha}} \\
& \quad=w_{2}^{12} s_{1}-\delta^{-1} w_{2}^{12} M_{2}^{1} v+\text { h.o.t. } \\
& (1+\alpha)^{2}\left(w_{1}^{12}\right)^{-1} s_{1}^{\alpha}\left[\left(w_{1}^{12}\right)^{-1} s_{1}^{1+\alpha}-\delta^{-1} M_{1}^{1} v+\text { h.o.t. }\right]^{\alpha} \\
& \quad=w_{2}^{12}+\text { h.o.t. }
\end{aligned}
$$

It is not difficult to see that (24) and (25) have a unique small positive solution

$$
s_{1}=\left(\frac{w_{1}^{12} M_{1}^{1} v}{(1+\alpha)^{2} M_{2}^{1} v}\right)^{1 / \alpha}+\text { h.o.t. }
$$

when $v \in R_{2}^{1}$ and $\left|M_{1}^{1} \nu\right|=o\left(\left|M_{2}^{1} \nu\right|^{1+\alpha}\right)$. Substituting (26) into (24), we get the surface $\mathrm{SN}^{2}$. It is easy to verify that the solution $s_{2}$ of (6) is positive as $s_{1}$ is given by (26) and $v \in \mathrm{SN}^{2}$. Thus, the proof of the lemma is complete.

Remark 4. Due to $N_{1}^{\prime \prime}\left(s_{1}\right)$, we see that the line $L_{1}\left(s_{1}\right)$ lies above the curve $N_{1}\left(s_{1}\right)$ as $v \in \mathrm{SN}^{2}$. Moreover, $L_{1}(0)=$ $-\delta^{-1} w_{2}^{12} M_{2}^{1} \nu+$ h.o.t. decreases as $\operatorname{rank}\left(M_{1}^{1}, M_{2}^{1}\right)=2$ and $\nu$ moves along the direction $-M_{2}^{1}$; in this case, system (1) exhibits two large 1-periodic orbits. And as $\nu$ leaves $\mathrm{SN}^{2}$ along the opposite direction, there is no large 1-periodic orbit.

Corollary 5. The bifurcation surface $\{0<\alpha \ll 1\} \times S N^{2}$ and the region $\left(\{0<\alpha \ll 1\} \times R_{2}^{1}\right) \cap\left\{(\alpha ; \nu):\left|M_{1}^{1} \nu\right|=\right.$ $\left.O\left(\left|M_{2}^{1} \nu\right|^{1+\alpha}\right), \alpha \ln \left|M_{2}^{1} \nu\right| \ll-1\right\}$ have no intersection point. 


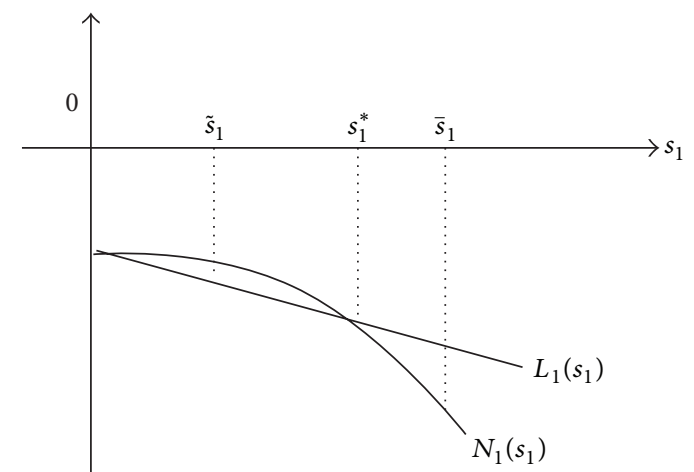

FIGURE 2: $N_{1}\left(s_{1}\right)$ and $L_{1}\left(s_{1}\right)$ for $0 \leq s_{1} \ll 1, v \in H_{2}^{1} \cap D_{2}$.

Proof. Let $w_{1}^{12}\left|M_{1}^{1} \nu\right|=-a^{\alpha}\left|M_{2}^{1} \nu\right|^{1+\alpha}$, where $0<a<\delta^{-1} e^{2}$ is a given constant; then, in view of $\left|M_{2}^{1} \nu\right|^{\alpha} \ll 1$ and $(1+\alpha)^{1 / \alpha} \rightarrow$ $e$ as $\alpha \rightarrow 0$, we know the right side of the expression $\mathrm{SN}^{2}$ as follows:

$$
\begin{aligned}
& {\left[\left(w_{1}^{12}\right)^{-1}\left(\frac{w_{1}^{12} M_{1}^{1} \nu}{(1+\alpha)^{2} M_{2}^{1} \nu}\right)^{(1+\alpha) / \alpha}-\delta^{-1} M_{1}^{1} \nu\right]^{1+\alpha}} \\
& -w_{2}^{12}\left(\frac{w_{1}^{12} M_{1}^{1} v}{(1+\alpha)^{2} M_{2}^{1} v}\right)^{1 / \alpha}+\text { h.o.t. } \\
& <\left[\frac{1}{4 w_{1}^{12} e^{2}} a^{1+\alpha}\left|M_{2}^{1} \nu\right|^{1+\alpha}-\frac{\delta^{-1}}{w_{1}^{12}} a^{\alpha}\left|M_{2}^{1} \nu\right|^{1+\alpha}\right]^{1+\alpha} \\
& -w_{2}^{12}\left(\frac{w_{1}^{12} M_{1}^{1} v}{(1+\alpha)^{2} M_{2}^{1} v}\right)^{1 / \alpha}+\text { h.o.t. } \\
& =\left(4 w_{1}^{12} e^{2}\right)^{-(1+\alpha)}\left|M_{2}^{1} \nu\right|^{(1+\alpha)^{2}}\left(a^{1+\alpha}-4 \delta^{-1} e^{2} a^{\alpha}\right)^{1+\alpha} \\
& -w_{2}^{12}\left(\frac{w_{1}^{12} M_{1}^{1} v}{(1+\alpha)^{2} M_{2}^{1} v}\right)^{1 / \alpha}+\text { h.o.t. } \\
& =a^{\alpha(1+\alpha)}\left(4 w_{1}^{12} e^{2}\right)^{-(1+\alpha)}\left|M_{2}^{1} \nu\right|^{(1+\alpha)^{2}}\left(a-4 \delta^{-1} e^{2}\right)^{1+\alpha} \\
& -w_{2}^{12} \frac{a}{e^{2}} M_{2}^{1} v+\text { h.o.t. } \\
& <\delta^{-1}\left|w_{2}^{12}\right| M_{2}^{1} v
\end{aligned}
$$

and $\delta^{-1}\left|w_{2}^{12}\right| M_{2}^{1} \nu$ is the left side of the expression $\mathrm{SN}^{2}$. Hence, the proof is complete.

Corollary 6. As $w_{1}^{12} w_{2}^{12}>1$, the bifurcation surface $\{0<\alpha \ll$ $1\} \times S N^{2}$ and the region $\left(\{0<\alpha \ll 1\} \times R_{2}^{1}\right) \cap\left\{(\alpha ; \nu):\left|M_{1}^{1} \nu\right|=\right.$ $\left.O\left(\left|M_{2}^{1} \nu\right|^{1+\alpha}\right),-1 \ll \alpha \ln \left|M_{2}^{1} \nu\right|<0\right\}$ have no intersection point.

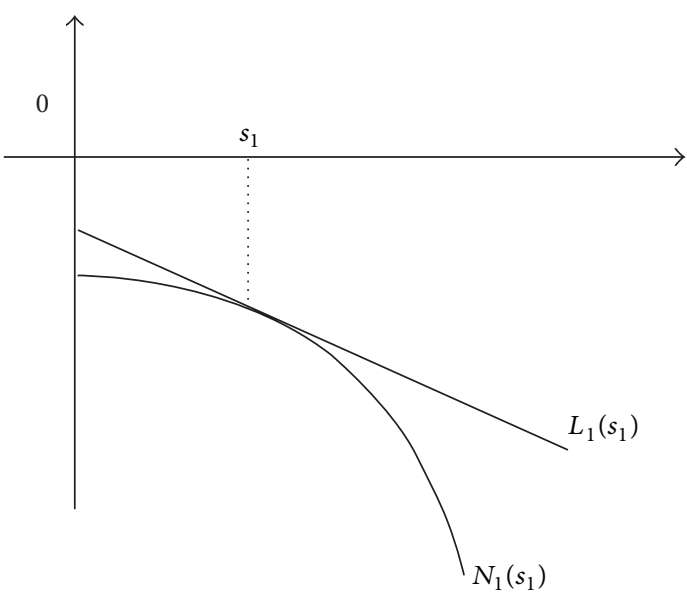

FIgURE 3: $N_{1}\left(s_{1}\right)$ and $L_{1}\left(s_{1}\right)$ for $0 \leq s_{1} \ll 1, v \in S N^{2}$.

Proof. Still let $w_{1}^{12}\left|M_{1}^{1} \nu\right|=-a^{\alpha}\left|M_{2}^{1} \nu\right|^{1+\alpha}$, where $0<a<\delta^{-1} e^{2}$ is a given constant; then, due to $\left|M_{2}^{1} \nu\right|^{\alpha}=1+o\left(\left|M_{2}^{1} \nu\right|\right)<1$, we know that

$$
\begin{aligned}
L \triangleq & -\delta^{-1} w_{1}^{12} w_{2}^{12} M_{2}^{1} \nu+w_{1}^{12} w_{2}^{12}\left(\frac{w_{1}^{12} M_{1}^{1} \nu}{(1+\alpha)^{2} M_{2}^{1} \nu}\right)^{1 / \alpha} \\
= & w_{1}^{12} w_{2}^{12}\left|M_{2}^{1} \nu\right|\left(-\delta^{-1}+a(1+\alpha)^{-2 / \alpha}\right) \\
R \triangleq & \left(w_{1}^{12}\right)^{-\alpha}\left[\left(\frac{w_{1}^{12} M_{1}^{1} \nu}{(1+\alpha)^{2} M_{2}^{1} \nu}\right)^{(1+\alpha) / \alpha}-\delta^{-1} w_{1}^{12}\left|M_{1}^{1} \nu\right|\right]^{1+\alpha} \\
& + \text { h.o.t. } \\
= & {\left[(1+\alpha)^{-2(1+\alpha) / \alpha} a^{1+\alpha}\left|M_{2}^{1} \nu\right|^{1+\alpha}-\delta^{-1} a^{\alpha}\left|M_{2}^{1} \nu\right|^{1+\alpha}\right]^{1+\alpha} } \\
& + \text { h.o.t. } \\
> & a^{\alpha(1+\alpha)}\left|M_{2}^{1} \nu\right|\left[\delta^{-1}+a(1+\alpha)^{-2 / \alpha}\right]^{1+\alpha}+\text { h.o.t. }
\end{aligned}
$$

As $w_{1}^{12} w_{2}^{12}>1, a>0$ is a constant, and $\alpha>0$ is small enough, it is easy to see that $R>L$. This contradicts the expression of $\mathrm{SN}^{2}$. The proof is complete.

By a similar analysis as in Lemmas 2 and 3, we can obtain the following results.

Lemma 7. Suppose that hypotheses $\left(H_{1}\right)-\left(H_{5}\right)$ and $0<\alpha \ll 1$ are valid; then, in addition to the large 1-homoclinic loop $\Gamma_{1}^{1}$, system (1) has exactly one simple large 1-periodic orbit near $\Gamma$ for $v \in H_{1}^{1} \cap D_{1}$. Moreover, the large 1-periodic orbit is persistent as $v$ changes in the neighborhood of $H_{1}^{1}$.

Lemma 8. Suppose that hypotheses $\left(H_{1}\right)-\left(H_{5}\right)$ and $0<\alpha \ll 1$ hold. Then, system (1) has a saddle-node bifurcation surface of 


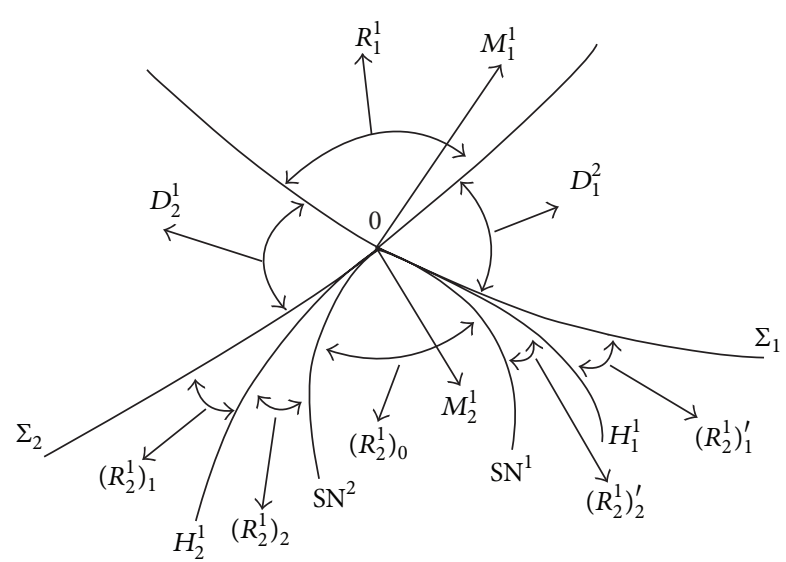

Figure 4: Bifurcation diagram in case $\Delta_{1}=\Delta_{2}=-1,0<\alpha \ll 1$, $-1 \ll \alpha \ln \left|M_{2}^{1} \nu\right|<0, w_{1}^{12} w_{2}^{12}<1$.

large 1-periodic orbit in the region $R_{1}^{1} \cap\left\{\nu:\left|M_{1}^{1} \nu\right|=o\left(\left|M_{2}^{1} \nu\right|\right\}\right.$ as follows:

$$
\begin{aligned}
S N^{1}: \delta^{-1} w_{1}^{12} M_{1}^{1} \nu= & {\left[\frac{\delta^{-1} M_{2}^{1} \nu(1+\alpha)^{2}}{(1+\alpha)^{2}-1}\right]^{1+\alpha} } \\
& -w_{1}^{12}\left(\frac{\delta^{-1} w_{2}^{12} M_{2}^{1} \nu}{(1+\alpha)^{2}-1}\right)^{1 /(1+\alpha)}+\text { h.o.t. }
\end{aligned}
$$

Remark 9. Let $N_{2}\left(s_{2}\right)=\left[\left(w_{2}^{12}\right)^{-1} s_{2}^{1+\alpha}+\delta^{-1} M_{2}^{1} \nu+\text { h.o.t. }\right]^{1+\alpha}$ and $L_{2}\left(s_{2}\right)=w_{1}^{12} s_{2}+\delta^{-1} w_{1}^{12} M_{1}^{1} v+$ h.o.t. Due to $N_{2}^{\prime \prime}\left(s_{2}\right)>0$ and $L_{2}(0)=\delta^{-1} w_{1}^{12} M_{1}^{1} v+$ h.o.t., we claim that the line $L_{2}\left(s_{2}\right)$ lies above the curve $N_{2}\left(s_{2}\right)$ as $\nu \in \mathrm{SN}^{1}$, and when $\operatorname{rank}\left(M_{1}^{1}, M_{2}^{1}\right)=2$ and $\nu$ leaves $\mathrm{SN}^{1}$ along the direction $M_{1}^{1}$, $L_{2}(0)$ decreases; hence, system (1) has two large 1-periodic orbits, and when $v$ moves along the direction $-M_{1}^{1}$, there is no large 1-periodic orbit.

Corollary 10. The bifurcation surface $\{0<\alpha \ll 1\} \times S N^{1}$ and the region $\left(\{0<\alpha \ll 1\} \times R_{2}^{1}\right) \cap\left\{(\alpha ; \nu):\left|M_{1}^{1} \nu\right|=\right.$ $\left.\mathrm{O}\left(\left|M_{2}^{1} \nu\right|^{1+\alpha}\right), \alpha \ln \left|M_{2}^{1} \nu\right| \ll-1\right\}$ have no intersection point.

Corollary 11. As $w_{1}^{12} w_{2}^{12}>1$, the bifurcation surface $\{0<\alpha \ll$ $1\} \times S N^{1}$ and the region $\left(\{0<\alpha \ll 1\} \times R_{2}^{1}\right) \cap\left\{(\alpha ; \nu):\left|M_{1}^{1} \nu\right|=\right.$ $\left.O\left(\left|M_{2}^{1} \nu\right|^{1+\alpha}\right),-1 \ll \alpha \ln \left|M_{2}^{1} \nu\right|<0\right\}$ have no intersection point.

In the following we define open regions in the neighborhood of the origin of the $\nu$-space, which are shown in Figures 4 and 5 .

$\left(R_{2}^{1}\right)_{0}$ is bounded by $\mathrm{SN}^{1}$ and $\mathrm{SN}^{2},\left(R_{2}^{1}\right)_{1}$ is bounded by $\Sigma_{2}$ and $H_{2}^{1},\left(R_{2}^{1}\right)_{2}$ is bounded by $H_{2}^{1}$ and $\mathrm{SN}^{2},\left(R_{2}^{1}\right)_{1}^{\prime}$ is bounded by $\mathrm{SN}^{1}$ and $\mathrm{SN}^{2},\left(R_{2}^{1}\right)_{2}^{\prime}$ is bounded by $\mathrm{SN}^{1}$ and $H_{1}^{1}, R_{1}^{1}$ is bounded by $\Sigma_{1}$ and $\Sigma_{2}$, and $\left(R_{2}^{1}\right)_{2}^{\prime \prime}$ is bounded by $H_{2}^{1}$ and $H_{1}^{1}$.

Now, the previous analysis is summarized in the following three theorems, as shown in Figures 4 and 5.

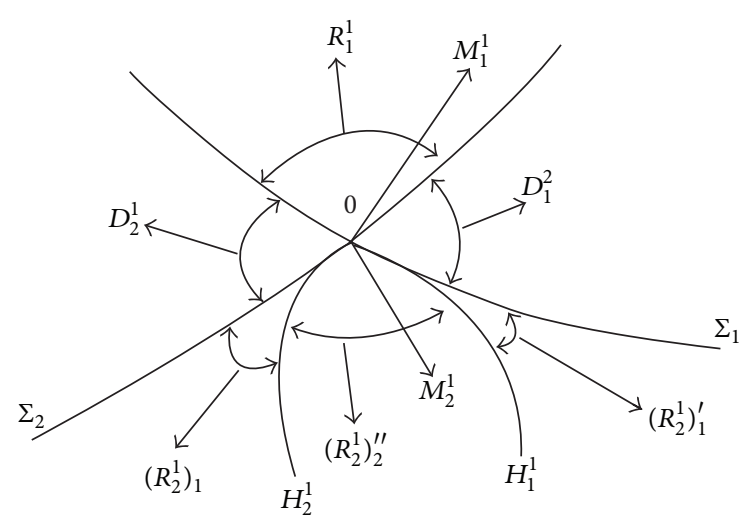

FIGURE 5: Bifurcation diagram in case $\Delta_{1}=\Delta_{2}=-1,0<\alpha \ll 1$, $-1 \ll \alpha \ln \left|M_{2}^{1} \nu\right|<0, w_{1}^{12} w_{2}^{12}>1$.

Theorem 12. Suppose that hypotheses $\left(H_{1}\right)-\left(H_{5}\right)$ hold, $0<$ $\alpha \ll 1,-1 \ll \alpha \ln \left|M_{2}^{1} \nu\right|<0, v \in R_{2}^{1}$, and $w_{1}^{12} w_{2}^{12}<1$. Then, system (1)

(1) has exactly one simple large 1-periodic orbit near $\Gamma$ as $\nu \in\left(R_{2}^{1}\right)_{1}$

(2) has a unique double large 1-periodic orbit near $\Gamma$ as $v \in$ $S N^{2}$

(3) has exactly two simple large 1-periodic orbits near $\Gamma$ as $\nu \in\left(R_{2}^{1}\right)_{2}$

(4) has exactly one simple large 1-periodic orbit and one large 1-homoclinic loop near $\Gamma$ as $v \in H_{2}^{1} \cap D_{2}$;

(5) does not have any large 1-periodic and large 1homoclinic loop near $\Gamma$ as $v \in\left(R_{2}^{1}\right)_{0}$;

(6) has exactly one simple large 1-periodic orbit near $\Gamma$ as $\nu \in\left(R_{2}^{1}\right)_{1}^{\prime}$

(7) has a unique double large 1-periodic orbit near $\Gamma$ as $v \in$ $S N^{1}$;

(8) has exactly two simple large 1-periodic orbits near $\Gamma$ as $\nu \in\left(R_{2}^{1}\right)_{2}^{\prime}$

(9) has exactly one simple large 1-periodic orbit and one large 1-homoclinic loop near $\Gamma$ as $\nu \in H_{1}^{1} \cap D_{1}$.

Theorem 13. Suppose that hypotheses $\left(H_{1}\right)-\left(H_{5}\right)$ hold, $0<$ $\alpha \ll 1,-1 \ll \alpha \ln \left|M_{2}^{1} \nu\right|<0, v \in R_{2}^{1}$, and $w_{1}^{12} w_{2}^{12}>1$. Then system (1)

(1) has exactly one simple large 1-periodic orbit near $\Gamma$ as $\nu \in\left(R_{2}^{1}\right)_{1}$

(2) has exactly one simple large 1-periodic orbit and one large 1-homoclinic loop near $\Gamma$ as $v \in H_{2}^{1} \cap D_{2}$;

(3) has exactly two simple large 1-periodic orbits near $\Gamma$ as $\nu \in\left(R_{2}^{1}\right)_{2}^{\prime \prime}$

(4) has exactly one simple large 1-periodic orbit and one large 1-homoclinic loop near $\Gamma$ as $v \in H_{1}^{1} \cap D_{1}$;

(5) has exactly one simple large 1-periodic orbit near $\Gamma$ as $\nu \in\left(R_{2}^{1}\right)_{1}^{\prime}$. 
Remark 14. Suppose that hypotheses $\left(H_{1}\right)-\left(H_{5}\right)$ hold, $0<$ $\alpha \ll 1, \alpha \ln \left|M_{2}^{1} \nu\right| \ll-1, \nu \in R_{2}^{1}$; then the conclusions of Theorem 13 are still true, and the bifurcation diagram is the same as Figure 5.

Denote by $D_{1}^{2}$ the open region with boundaries $\Sigma_{1}$ and $\Sigma_{2}$, such that $D_{1}^{2} \cap\left\{v: M_{1}^{1} v>0, M_{2}^{1} v>0,|v| \ll 1\right\} \neq \emptyset$. $D_{2}^{1}$ is the open region with boundaries $\Sigma_{2}$ and $\Sigma_{1}$, such that $D_{2}^{1} \cap\left\{\nu: M_{1}^{1} \nu<0, M_{2}^{1} \nu<0,|\nu| \ll 1\right\} \neq \emptyset$.

From the bifurcation equations (6), we have the following theorem.

Theorem 15. Suppose that hypotheses $\left(H_{1}\right)-\left(H_{5}\right)$ hold, and $0<\alpha \ll 1$. Then, system (1)

(1) does not have any large 1-periodic orbit near $\Gamma$ as $\nu \in$ $D_{1}^{2}$;

(2) does not have any large 1-periodic orbit near $\Gamma$ as $\nu \in$ $D_{2}^{1}$;

(3) does not have any large 1-periodic orbit near $\Gamma$ as $\nu \in$ $R_{1}^{1}$.

Remark 16. For $-1 \ll \alpha<0$, by the same analysis, we can obtain the analogous results as those in Theorems 1215. In fact, in the case we can reverse the time $t$ and change $(x, y, u, v)$ to $(y, x, v, u)$, and then we still get $0<\alpha \ll 1$.

At last, we consider the case $\alpha=0$. By a similar process to that of Section 3, we obtain the bifurcation equations

$$
\begin{gathered}
-\left(w_{1}^{12}\right)^{-1} s_{1}+s_{2}+\delta^{-1} M_{1}^{1} v+\text { h.o.t. }=0 \\
\left(w_{2}^{12}\right)^{-1} s_{2}-s_{1}+\delta^{-1} M_{2}^{1} v+\text { h.o.t. }=0 .
\end{gathered}
$$

For the same reason as before, we have the following theorem.

Theorem 17. Suppose that $\left(H_{1}\right)-\left(H_{5}\right), \quad \alpha=0$, and $\operatorname{rank}\left(M_{1}^{1}, M_{2}^{1}\right)=2$ hold; then,

(1) as $v \in R_{2}^{1}$, system (1) not only has a large 1-homoclinic orbit bifurcation surface

$$
w_{2}^{12} M_{2}^{1} v-M_{1}^{1} v+\text { h.o.t. }=0
$$

with a normal vector $\left(w_{2}^{12} M_{2}^{1}-M_{1}^{1}\right)$ at $v=0$, but also has another large 1-homoclinic orbit bifurcation surface

$$
w_{1}^{12} M_{1}^{1} v-M_{2}^{1} v+\text { h.o.t. }=0
$$

with a normal vector $\left(w_{1}^{12} M_{1}^{1}-M_{2}^{1}\right)$ at $v=0$,

(2) as either $w_{1}^{12} w_{2}^{12}<1, M_{2}^{1} v>w_{1}^{12} M_{1}^{1} v$, and $w_{2}^{12} M_{2}^{1} v>$ $M_{1}^{1} v$ or $w_{1}^{12} w_{2}^{12}>1, M_{2}^{1} \nu<w_{1}^{12} M_{1}^{1} v$, and $w_{2}^{12} M_{2}^{1} \nu<$ $M_{1}^{1} v$, system (1) has a unique large 1-periodic orbit near $\Gamma$.
Case $2\left(\Delta_{1}=-1, \Delta_{2}=1\right.$ (i.e., single twisted)). Similar to Case 1, for convenience and simplicity, we use the following notations:

$\bar{R}_{1}^{1}=\left\{v: M_{1}^{1} v<0, M_{2}^{1} v>0,\left|M_{2}^{1} v\right|=O\left(\left|M_{1}^{1} v\right|\right),|v| \ll 1\right\}$,

$\bar{R}_{2}^{1}=\left\{v: M_{1}^{1} v<0, M_{2}^{1} v<0,\left|M_{1}^{1} v\right|=O\left(\left|M_{2}^{1} v\right|\right),|v| \ll 1\right\}$,

$\bar{D}_{1}^{2}=\left\{v: M_{1}^{1} v>0, M_{2}^{1} v>0,|v| \ll 1\right\}$,

$\bar{D}_{2}^{1}=\left\{\nu: M_{1}^{1} v>0, M_{2}^{1} \nu<0,|v| \ll 1\right\}$.

If (6) has a solution $s_{1}=s_{2}=0$, then we have

$$
M_{i}^{1} \nu+\text { h.o.t. }=0, \quad i=1,2 \text {. }
$$

If $M_{i}^{1} \neq 0$, then there exists a codimension 1 surface $\bar{\Sigma}_{i}$ with a normal vector $M_{i}^{1}$ at $\nu=0$, such that the $i$ th equation of (6) has solution $s_{1}=s_{2}=0$ as $\nu \in \bar{\Sigma}_{i}$ and $|\nu| \ll 1$; that is, $\Gamma_{i}$ is persistent. If $\operatorname{rank}\left(M_{1}^{1}, M_{2}^{1}\right)=2$, then $\bar{\Sigma}_{12}=\bar{\Sigma}_{1} \cap \bar{\Sigma}_{2}$ is a codimension 2 surface with a normal plane $\operatorname{span}\left\{M_{1}^{1}, M_{2}^{1}\right\}$ such that (6) has solution $s_{1}=s_{2}=0$ as $\nu \in \bar{\Sigma}_{12}$ and $|\nu| \ll 1$; equivalently, the large loop $\Gamma=\Gamma_{1} \cup \Gamma_{2}$ is persistent.

Suppose that (6) has solution $s_{1}=0, s_{2}>0$. Then, we have

$$
\begin{gathered}
s_{2}=-\delta^{-1} M_{1}^{1} v+\text { h.o.t., } \\
0=\left(w_{2}^{12}\right)^{-1} s_{2}^{1+\alpha}+\delta^{-1} M_{2}^{1} v+\text { h.o.t. }
\end{gathered}
$$

Hence, we get the large 1-homoclinic orbit bifurcation surface equation

$$
\bar{H}_{2}^{1}: \delta^{-1} w_{2}^{12} M_{2}^{1} \nu+\left(-\delta^{-1} M_{1}^{1} v\right)^{1+\alpha}+\text { h.o.t. }=0,
$$

which is well defined at least in the region $\bar{R}_{2}^{1}$ and has a normal vector $M_{2}^{1}$ as $\alpha>0$ (resp., $M_{1}^{1}$ as $\alpha<0$ ) at $\nu=0$ such that the system (1) has a large 1-homoclinic loop $\Gamma_{2}^{1}$ near $\Gamma$ as $v \in \bar{H}_{2}^{1}$. It also means that $\bar{H}_{2}^{1}$ is tangent to $\bar{\Sigma}_{2}$ as $\alpha>0$ (resp., $\bar{\Sigma}_{1}$ as $\alpha<$ $0)$. When $v \in \bar{H}_{2}^{1}, \alpha>0, s_{1}=0$, and $s_{2}=-\delta^{-1} M_{1}^{1} v+$ h.o.t., (6) implies that

$$
\begin{gathered}
s_{2 v}=-\delta^{-1} M_{1}^{1}+\text { h.o.t. } \\
s_{1 v}=(1+\alpha)\left(w_{2}^{12}\right)^{-1}\left(-\delta^{-1} M_{1}^{1} \nu\right)^{\alpha} s_{2 v}+\delta^{-1} M_{2}^{1}+\text { h.o.t. }
\end{gathered}
$$

Using (37) $\times(1+\alpha)\left(w_{2}^{12}\right)^{-1}\left(-\delta^{-1} M_{1}^{1} \nu\right)^{\alpha}+(38)$, we get

$$
\left.s_{1 \nu}\right|_{\bar{H}_{2}^{1}}=\delta^{-1} M_{2}^{1}+O\left(\left|M_{1}^{1} \nu\right|^{\alpha}\right) \text {. }
$$

So, $s_{1}=s_{1}(\nu)$ increases along the direction of the gradient $M_{2}^{1}$ for $v$ near $\bar{H}_{2}^{1}$.

Similarly, by setting $s_{k}=e^{-\rho_{1} \tau_{k}}$, we can derive that $s_{1}(\nu)$ increases along the direction $-M_{1}^{1}$ for near $\bar{H}_{2}^{1}$ as $\alpha>0$. 
If (6) has solution $s_{1}>0$ and $s_{2}=0$, then we get

$$
\begin{gathered}
0=\left(w_{1}^{12}\right)^{-1} s_{1}^{1+\alpha}-\delta^{-1} M_{1}^{1} v+\text { h.o.t. } \\
s_{1}=\delta^{-1} M_{2}^{1} v+\text { h.o.t. }
\end{gathered}
$$

Therefore, we obtain the existence of the other large 1homoclinic orbit bifurcation surface equation

$$
\bar{H}_{1}^{1}: \delta^{-1} w_{1}^{12} M_{1}^{1} \nu+\left(\delta^{-1} M_{2}^{1} v\right)^{1+\alpha}+\text { h.o.t. }=0,
$$

which is well defined at least in the region $\bar{R}_{1}^{1}$ and has a normal vector $M_{1}^{1}$ as $\alpha>0$ (resp., $M_{2}^{1}$ as $\alpha<0$ ) at $\nu=0$ such that the system (1) has a large 1-homoclinic loop $\Gamma_{1}^{1}$ near $\Gamma$ as $v \in \bar{H}_{1}^{1}$. Thus, $\bar{H}_{1}^{1}$ is also tangent to $\bar{\Sigma}_{1}$ as $\alpha>0$ (resp., $\bar{\Sigma}_{2}$ as $\alpha<0$ ). For $v \in \bar{H}_{1}^{1}$ and $\alpha>0, s_{1}=\delta^{-1} M_{2}^{1} v+$ h.o.t., $s_{2}=0$, it follows from (6) that

$$
\begin{gathered}
s_{2 v}=(1+\alpha)\left(w_{1}^{12}\right)^{-1}\left(\delta^{-1} M_{2}^{1} \nu\right)^{\alpha} s_{1 v}-\delta^{-1} M_{1}^{1}+\text { h.o.t. } \\
s_{1 v}=\delta^{-1} M_{2}^{1}+\text { h.o.t. }
\end{gathered}
$$

Computing $(43) \times(1+\alpha)\left(w_{1}^{12}\right)^{-1}\left(\delta^{-1} M_{2}^{1} \nu\right)^{\alpha}+(42)$, it leads to

$$
\left.s_{2 \nu}\right|_{\bar{H}_{1}^{1}}=-\delta^{-1} M_{1}^{1}+O\left(\left|M_{2}^{1} \nu\right|^{\alpha}\right) .
$$

So, $s_{2}=s_{2}(\nu)$ increases along the direction $-M_{1}^{1}$ for $\nu$ close to $\bar{H}_{1}^{1}$.

By a similar procedure, we can show that $s_{2}(\nu)$ increases along the direction $M_{2}^{1}$ as $\nu$ is in the neighborhood of $\bar{H}_{1}^{1}$ and $\alpha<0$.

Summarizing the previous analysis, we have the following theorem.

Theorem 18. Suppose that $\left(H_{1}\right)-\left(H_{5}\right)$ hold; then, the following conclusions are true.

(1) If $M_{i}^{1} \neq 0$, then there exists a unique surface $\bar{\Sigma}_{i}$ with codimension 1 and normal vectors $M_{i}^{1}$ at $v=0$, such that system (1) has a homoclinic loop near $\Gamma_{i}$ if and only if $\nu \in \bar{\Sigma}_{i}$ and $|\nu| \ll 1$. If $\operatorname{rank}\left(M_{1}^{1}, M_{2}^{1}\right)=2$, then $\bar{\Sigma}_{12}=\bar{\Sigma}_{1} \cap \bar{\Sigma}_{2}$ is a codimension 2 surface and $0 \in \bar{\Sigma}_{12}$ such that system (1) has a large loop consisting of two homoclinic orbits near $\Gamma$ as $\nu \in \bar{\Sigma}_{12}$ and $|\nu| \ll 1$; that is, $\Gamma$ is persistent.

(2) In the region $\bar{R}_{2}^{1}$, there exists a unique large 1-homoclinic orbit bifurcation surface $\bar{H}_{2}^{1}$ which is tangent to $\bar{\Sigma}_{2}$ (resp., $\bar{\Sigma}_{1}$ ) at $v=0$ with the normal vector $M_{2}^{1}$ (resp., $M_{1}^{1}$ ) as $\alpha>0$ (resp., $\alpha<0$ ), and for $\nu \in \bar{H}_{2}^{1}$, system (1) has a unique large 1-homoclinic orbit near $\Gamma$. Furthermore, the unique large 1-homoclinic orbit near $\Gamma$ becomes a large 1-periodic orbit when $\nu$ moves along the direction $M_{2}^{1}$ (resp., $-M_{1}^{1}$ ) and nearby $\bar{H}_{2}^{1}$ as $\alpha>0$ (resp., $\alpha<0$ ). In the region $\bar{R}_{1}^{1}$, there exists another unique large 1-homoclinic orbit bifurcation surface $\bar{H}_{1}^{1}$ which is tangent to $\bar{\Sigma}_{1}$ (resp., $\bar{\Sigma}_{2}$ ) at $v=0$ with the normal $M_{1}^{1}$ (resp., $M_{2}^{1}$ ) as $\alpha>0$ (resp., $\alpha<0$ ), and for $v \in \bar{H}_{1}^{1}$, system (1) has another one unique large 1homoclinic orbit near $\Gamma$. Furthermore, the unique large 1-homoclinic orbit near $\Gamma$ changes into a large 1-periodic orbit when $v$ shifts along the direction $-M_{1}^{1}$ (resp., $M_{2}^{1}$ ) and nearby $\bar{H}_{1}^{1}$ as $\alpha>0$ (resp., $\left.\alpha<0\right)$.

Lemma 19. Suppose that hypotheses $\left(H_{1}\right)-\left(H_{5}\right)$ and $0<\alpha \ll$ 1 are valid; system (1) has only a unique large 1-homoclinic orbit $\Gamma_{2}^{1}$ near $\Gamma$ for $\nu \in \bar{H}_{2}^{1}$ and $0<|\nu| \ll 1$.

Proof. If $\nu \in \bar{H}_{2}^{1}$ and $|\nu| \ll 1$, from Theorem 18, we know that system (1) has one large 1-homoclinic loop $\Gamma_{2}^{1}$. Now, following (6), we have

$$
\begin{aligned}
& {\left[\left(w_{1}^{12}\right)^{-1} s_{1}^{1+\alpha}-\delta^{-1} M_{1}^{1} \nu+\text { h.o.t. }\right]^{1+\alpha}=w_{2}^{12} s_{1}-\delta^{-1} w_{2}^{12} M_{2}^{1} \nu} \\
& + \text { h.o.t. }
\end{aligned}
$$

Let $N_{1}\left(s_{1}\right)$ and $L_{1}\left(s_{1}\right)$ be the left- and right-hand sides of (45), respectively. Then, by (36), we get $N_{1}(0)=L_{1}(0)$ as $v \in \bar{H}_{2}^{1}$. Moreover,

$$
\begin{gathered}
N_{1}^{\prime}\left(s_{1}\right)=(1+\alpha)^{2}\left(w_{1}^{12}\right)^{-1}\left[\left(w_{1}^{12}\right)^{-1} s_{1}^{1+\alpha}-\delta^{-1} M_{1}^{1} \nu\right. \\
\quad+\text { h.o.t. }]^{\alpha} s_{1}^{\alpha}, \\
L_{1}^{\prime}\left(s_{1}\right)=w_{2}^{12}+\text { h.o.t. }
\end{gathered}
$$

Thus, $N_{1}^{\prime}\left(s_{1}\right) \leq 0<L_{1}^{\prime}\left(s_{1}\right)$, and $N_{1}^{\prime \prime}\left(s_{1}\right)<0$. Therefore, $N_{1}\left(s_{1}\right)<L_{1}\left(s_{1}\right)$ for $s_{1} \neq 0$, as shown in Figure 6 .

Therefore, system (1) has only a unique large 1-homoclinic orbit near $\Gamma$ for $\nu \in \bar{H}_{2}^{1}$ and $0<|\nu| \ll 1$.

Remark 20. Due to $N_{1}^{\prime \prime}\left(s_{1}\right)<0$, we can see that the line $N_{1}\left(s_{1}\right)$ lies under the curve $L_{1}\left(s_{1}\right)$ as $v \in \bar{H}_{2}^{1}$. Moreover, $L_{1}(0)=$ $-\delta^{-1} w_{2}^{12} M_{2}^{1} v+$ h.o.t. decreases as $\operatorname{rank}\left(M_{1}^{1}, M_{2}^{1}\right)=2$ and $v$ moves along the direction $M_{2}^{1}$; in this case, system (1) has a unique large 1-periodic orbit. And as $v$ leaves $\bar{H}_{2}^{1}$ along the opposite direction, there is no large 1-periodic orbit.

By a similar analysis as in Lemma 19 , we can obtain the following results.

Lemma 21. Suppose that hypotheses $\left(H_{1}\right)-\left(H_{5}\right)$ and $0<\alpha \ll$ 1 are valid; system (1) has only a unique large 1-homoclinic orbit $\Gamma_{1}^{1}$ near $\Gamma$ for $\nu \in \bar{H}_{1}^{1}$ and $0<|\nu| \ll 1$.

Remark 22. Let $N_{2}\left(s_{2}\right)=\left[\left(w_{2}^{12}\right)^{-1} s_{2}^{1+\alpha}+\delta^{-1} M_{2}^{1} \nu+\text { h.o.t. }\right]^{1+\alpha}$ and $L_{2}\left(s_{2}\right)=w_{1}^{12} s_{2}+\delta^{-1} w_{1}^{12} M_{1}^{1} v+$ h.o.t. Due to $N_{2}^{\prime \prime}\left(s_{2}\right)>0$ and $L_{2}(0)=\delta^{-1} w_{1}^{12} M_{1}^{1} \nu+$ h.o.t., we claim that the line 


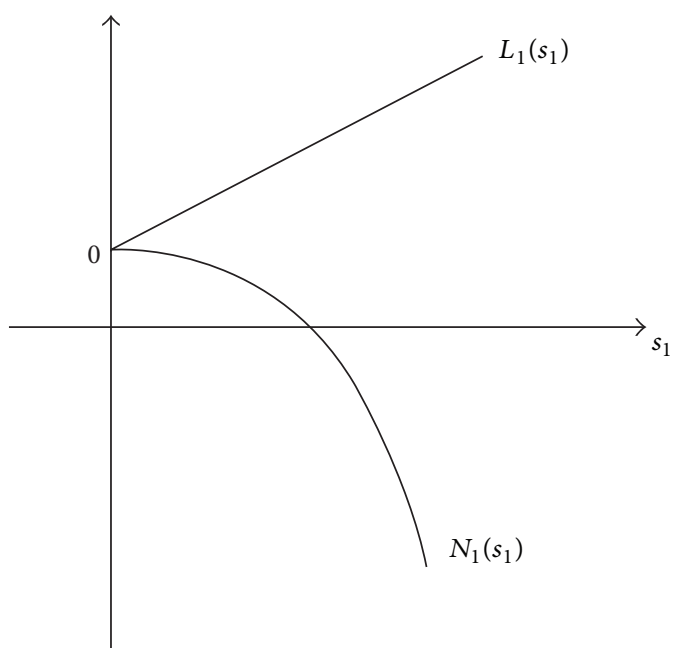

FIGURE 6: $N_{1}\left(s_{1}\right)$ and $L_{1}\left(s_{1}\right)$ for $0 \leq s_{1} \ll 1, v \in \bar{H}_{2}^{1}$.

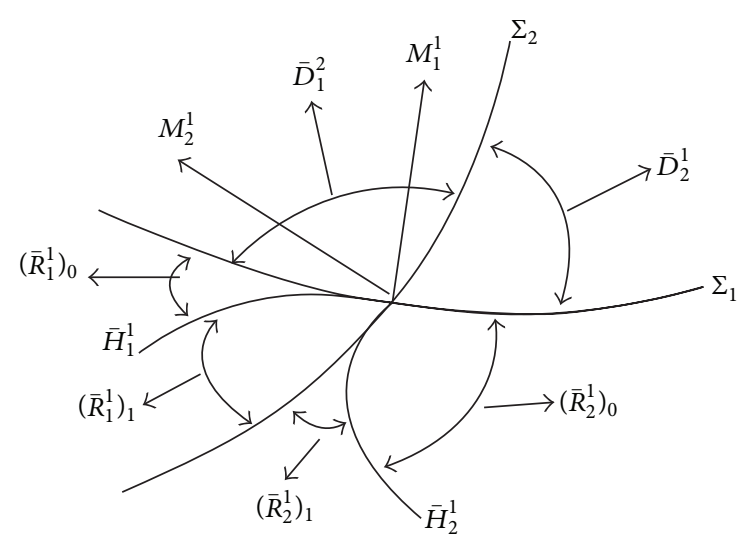

FIgURE 7: Bifurcation diagram in case $\Delta_{1}=-1, \Delta_{2}=1,0<\alpha \ll 1$.

$L_{2}\left(s_{2}\right)$ lies under the curve $N_{2}\left(s_{2}\right)$ as $v \in \bar{H}_{1}^{1}$, and when $\operatorname{rank}\left(M_{1}^{1}, M_{2}^{1}\right)=2$ and $v$ leaves $\bar{H}_{1}^{1}$ along the direction $-M_{1}^{1}, L_{2}(0)$ increases; hence, system (1) has a unique large 1-periodic orbit, and when $v$ moves along the direction $M_{1}^{1}$, there is no large 1-periodic orbit.

In the following we define open regions in the neighborhood of the origin of the $v$-space, which are shown in Figure 7.

$\left(\bar{R}_{2}^{1}\right)_{0}$ is bounded by $\Sigma_{1}$ and $\bar{H}_{2}^{1},\left(\bar{R}_{2}^{1}\right)_{1}$ is bounded by $\Sigma_{2}$ and $\bar{H}_{2}^{1},\left(\bar{R}_{1}^{1}\right)_{1}$ is bounded by $\bar{H}_{1}^{1}$ and $\Sigma_{2},\left(\bar{R}_{1}^{1}\right)_{0}$ is bounded by $\Sigma_{1}$ and $\bar{H}_{1}^{1}, \bar{D}_{2}^{1}$ is bounded by $\Sigma_{1}$ and $\Sigma_{2}$, and $\bar{D}_{1}^{2}$ is bounded by $\Sigma_{2}$ and $\Sigma_{1}$.

From the previous analysis, we obtain the following three theorems, as shown in Figure 7.

Theorem 23. Suppose that hypotheses $\left(H_{1}\right)-\left(H_{5}\right)$ hold, $0<$ $\alpha \ll 1, v \in \bar{R}_{2}^{1}$. Then, system (1)

(1) does not have any large 1-periodic orbit and large 1homoclinic loop near $\Gamma$ as $v \in\left(\bar{R}_{2}^{1}\right)_{0}$;
(2) has a unique large 1-homoclinic loop near $\Gamma$ as $v \in \bar{H}_{2}^{1}$;

(3) has a unique large 1-periodic orbit near $\Gamma$ as $v \in\left(\bar{R}_{2}^{1}\right)_{1}$.

Theorem 24. Suppose that hypotheses $\left(H_{1}\right)-\left(H_{5}\right)$ hold, $0<$ $\alpha \ll 1, v \in \bar{R}_{1}^{1}$. Then, system (1)

(1) has no large 1-periodic orbit and large 1-homoclinic loop near $\Gamma$ as $\nu \in\left(\bar{R}_{1}^{1}\right)_{0}$;

(2) has a unique large 1-homoclinic loop near $\Gamma$ as $v \in \bar{H}_{1}^{1}$;

(3) has a unique large 1-periodic orbit near $\Gamma$ as $\nu \in\left(\bar{R}_{1}^{1}\right)_{1}$.

From the bifurcation equations, we have the following theorem easily.

Theorem 25. Suppose that hypotheses $\left(H_{1}\right)-\left(H_{5}\right)$ hold, and $0<\alpha \ll 1$. Then, system (1)

(1) has no large 1-periodic orbit near $\Gamma$ as $v \in \bar{D}_{1}^{2}$;

(2) has no large 1-periodic orbit near $\Gamma$ as $\nu \in \bar{D}_{2}^{1}$.

Remark 26. For $-1 \ll \alpha<0$, by the same analysis, we can obtain the analogous results as those in Theorems 2325. In fact, in the case we can reverse the time $t$ and change $(x, y, u, v)$ to $(y, x, v, u)$, and then we still get $0<\alpha \ll 1$.

At last, we consider the case $\alpha=0$. By a similar process to that of Section 3, we obtain the bifurcation equations

$$
\begin{gathered}
-\left(w_{1}^{12}\right)^{-1} s_{1}+s_{2}+\delta^{-1} M_{1}^{1} v+\text { h.o.t. }=0, \\
\left(w_{2}^{12}\right)^{-1} s_{2}-s_{1}+\delta^{-1} M_{2}^{1} v+\text { h.o.t. }=0 .
\end{gathered}
$$

For the same reason as before, we have the following theorem.

Theorem 27. Suppose that $\left(H_{1}\right)-\left(H_{5}\right), \quad \alpha=0, \quad$ and $\operatorname{rank}\left(M_{1}^{1}, M_{2}^{1}\right)=2$ hold; then,

(1) as $v \in \bar{R}_{2}^{1}$, system (1) has a unique large 1-homoclinic orbit bifurcation surface

$$
w_{2}^{12} M_{2}^{1} v-M_{1}^{1} v+\text { h.o.t. }=0
$$

with a normal vector $\left(w_{2}^{12} M_{2}^{1}-M_{1}^{1}\right)$ at $v=0$;

(2) as $v \in \bar{R}_{1}^{1}$, system (1) also has a unique large 1-homoclinic orbit bifurcation surface

$$
w_{1}^{12} M_{1}^{1} v-M_{2}^{1} v+\text { h.o.t. }=0
$$

with a normal vector $\left(w_{1}^{12} M_{1}^{1}-M_{2}^{1}\right)$ at $v=0$;

(3) as $M_{2}^{1} v<w_{1}^{12} M_{1}^{1} v$ and $w_{2}^{12} M_{2}^{1} v>M_{1}^{1} v$, system (1) has a unique large 1-periodic orbit near $\Gamma$.

Remark 28. As $\Gamma_{1}$ is nontwisted and $\Gamma_{2}$ is twisted, we can obtain similar conclusions as shown in Case 2. 


\section{Conclusion}

This paper is devoted to investigating the twisting bifurcations of double homoclinic loops with resonant eigenvalues in 4-dimensional systems. We give asymptotic expressions of the bifurcation surfaces and their relative positions, describe the existence regions of large 1-periodic orbits near $\Gamma$ in Lemmas 2 and 7, and obtain the sufficient conditions for the existence or nonexistence of saddle-node bifurcation surfaces in Lemmas 3 and 8. More importantly, the complete bifurcation diagrams are given under different cases in Figures 4, 5, and 7. According to our analysis, when $\Gamma$ is double twisted, in Theorems 12-15, we obtain one bifurcation diagram with saddle-node bifurcation surfaces of large 1-periodic orbit when $w_{1}^{12} w_{2}^{12}<1$ and the other bifurcation diagram without saddle-node bifurcation surfaces when $w_{1}^{12} w_{2}^{12}>1$. When $\Gamma$ is single twisted, in Theorems 23-25, we obtain another bifurcation diagram. Compared with the nontwisted cases in Zhang et al. [32], our paper shows completely different results and bifurcation diagrams. It is worthy to be mentioned that the restriction on the dimension is not essential, the method used in this paper can be extended to higher dimensional systems without any difficulty, and the same conclusions can be deduced under the same hypotheses. Furthermore, we mention some problems for future study. (1) $W^{s}$ or $W^{u}$ is inclination flip on one of the double homoclinic loops. (2) Both $W^{s}$ and $W^{u}$ are inclination flips on the double homoclinic loops and so on. But the difficulty of these problems will increase with adding codimension of the double homoclinic loops.

\section{Acknowledgments}

The research was supported by National Natural Science Foundation of China (nos. 11001041, 11101170, 11202192, 11271065 and 10926105), SRFDP (no. 200802001008), and the State Scholarship Fund of the China Scholarship Council (nos. 2011662521, and 2011842509).

\section{References}

[1] D. Blázquez-Sanz and K. Yagasaki, "Analytic and algebraic conditions for bifurcations of homoclinic orbits I: saddle equilibria," Journal of Differential Equations, vol. 253, no. 11, pp. 2916-2950, 2012.

[2] S.-N. Chow, B. Deng, and B. Fiedler, "Homoclinic bifurcation at resonant eigenvalues," Journal of Dynamics and Differential Equations, vol. 2, no. 2, pp. 177-244, 1990.

[3] S.-N. Chow and X.-B. Lin, "Bifurcation of a homoclinic orbit with a saddle-node equilibrium," Differential and Integral Equations, vol. 3, no. 3, pp. 435-466, 1990.

[4] B. Deng, "Homoclinic twisting bifurcations and cusp horseshoe maps," Journal of Dynamics and Differential Equations, vol. 5, no. 3, pp. 417-467, 1993.

[5] J. Gruendler, "Homoclinic solutions for autonomous dynamical systems in arbitrary dimension," SIAM Journal on Mathematical Analysis, vol. 23, no. 3, pp. 702-721, 1992.
[6] J. Gruendler, "Homoclinic solutions for autonomous ordinary differential equations with nonautonomous perturbations," Journal of Differential Equations, vol. 122, no. 1, pp. 1-26, 1995.

[7] A. J. Homburg, "Global aspects of homoclinic bifurcations of vector fields," Memoirs of the American Mathematical Society, vol. 121 , no. $578,1996$.

[8] A. J. Homburg and B. Krauskopf, "Resonant homoclinic flip bifurcations," Journal of Dynamics and Differential Equations, vol. 12, no. 4, pp. 807-850, 2000.

[9] Y. L. Jin and D. M. Zhu, "Bifurcations of rough heteroclinic loop with two saddle points," Science in China A, vol. 46, no. 4, pp. 459-468, 2003.

[10] M. Kisaka, H. Kokubu, and H. Oka, "Bifurcations to $n$ homoclinic orbits and $n$-periodic orbits in vector fields," Journal of Dynamics and Differential Equations, vol. 5, no. 2, pp. 305-357, 1993.

[11] H. Kokubu, M. Komuro, and H. Oka, "Multiple homoclinic bifurcations from orbit-flip. I. Successive homoclinic doublings," International Journal of Bifurcation and Chaos in Applied Sciences and Engineering, vol. 6, no. 5, pp. 833-850, 1996.

[12] J. Knobloch and T. Wagenknecht, "Homoclinic snaking near a heteroclinic cycle in reversible systems," Physica D, vol. 206, no. 1-2, pp. 82-93, 2005.

[13] X. Liu and D. Zhu, "Bifurcation of degenerate homoclinic orbits to saddle-center in reversible systems," Chinese Annals of Mathematics. Series B, vol. 29, no. 6, pp. 575-584, 2008.

[14] B. E. Oldeman, B. Krauskopf, and A. R. Champneys, "Numerical unfoldings of codimension-three resonant homoclinic flip bifurcations," Nonlinearity, vol. 14, no. 3, pp. 597-621, 2001.

[15] B. Sandstede, "Constructing dynamical systems having homoclinic bifurcation points of codimension two," Journal of Dynamics and Differential Equations, vol. 9, no. 2, pp. 269-288, 1997.

[16] S. Schecter and C. Sourdis, "Heteroclinic orbits in slow-fast Hamiltonian systems with slow manifold bifurcations," Journal of Dynamics and Differential Equations, vol. 22, no. 4, pp. 629$655,2010$.

[17] S. L. Shui and D. M. Zhu, "Codimension 3 nonresonant bifurcations of homoclinic orbits with two inclination flips," Science in China A, vol. 48, no. 2, pp. 248-260, 2005.

[18] T. Wagenknecht, "Two-heteroclinic orbits emerging in the reversible homoclinic pitchfork bifurcation," Nonlinearity, vol. 18, no. 2, pp. 527-542, 2005.

[19] S. Wiggins, Global Bifurcations and Chaos, vol. 73, Springer, New York, NY, USA, 1988.

[20] S. Wiggins, Introduction to Applied Nonlinear Dynamical Systems and Chaos, vol. 2, Springer, New York, NY, USA, 1990.

[21] T. Zhang and D. Zhu, "Codimension 3 homoclinic bifurcation of orbit flip with resonant eigenvalues corresponding to the tangent directions," International Journal of Bifurcation and Chaos in Applied Sciences and Engineering, vol. 14, no. 12, pp. 4161-4175, 2004.

[22] D. M. Zhu, "Problems in homoclinic bifurcation with higher dimensions," Acta Mathematica Sinica, vol. 14, no. 3, pp. 341352, 1998.

[23] D. Zhu and Z. Xia, "Bifurcations of heteroclinic loops," Science in China A, vol. 41, no. 8, pp. 837-848, 1998.

[24] M. Han and P. Bi, "Bifurcation of limit cycles from a double homoclinic loop with a rough saddle," Chinese Annals of Mathematics B, vol. 25, no. 2, pp. 233-242, 2004. 
[25] M. A. Han and J. Chen, "On the number of limit cycles in double homoclinic bifurcations," Science in China A, vol. 43, no. 9, pp. 914-928, 2000.

[26] A. J. Homburg and J. Knobloch, "Multiple homoclinic orbits in conservative and reversible systems," Transactions of the American Mathematical Society, vol. 358, no. 4, pp. 1715-1740, 2006.

[27] C. A. Morales, M. J. Pacifico, and B. San Martin, "Expanding Lorenz attractors through resonant double homoclinic loops," SIAM Journal on Mathematical Analysis, vol. 36, no. 6, pp. 18361861, 2005.

[28] C. A. Morales, M. J. Pacifico, and B. San Martin, "Contracting Lorenz attractors through resonant double homoclinic loops," SIAM Journal on Mathematical Analysis, vol. 38, no. 1, pp. 309332, 2006.

[29] Q. Lu, "Codimension 2 bifurcation of twisted double homoclinic loops," Computers \& Mathematics with Applications, vol. 57, no. 7, pp. 1127-1141, 2009.

[30] C. G. Ragazzo, "On the stability of double homoclinic loops," Communications in Mathematical Physics, vol. 184, no. 2, pp. 251-272, 1997.

[31] W. P. Zhang and D. M. Zhu, "Codimension 2 bifurcations of double homoclinic loops," Chaos, Solitons and Fractals, vol. 39, no. 1, pp. 295-303, 2009.

[32] W. Zhang, D. Zhu, and D. Liu, "Codimension 3 nontwisted double homoclinic loops bifurcations with resonant eigenvalues," Journal of Dynamics and Differential Equations, vol. 20, no. 4, pp. 893-908, 2008. 


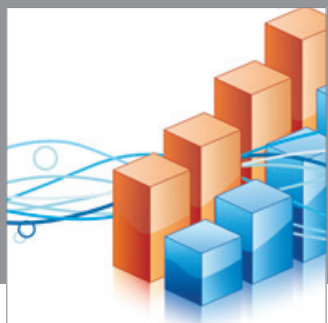

Advances in

Operations Research

mansans

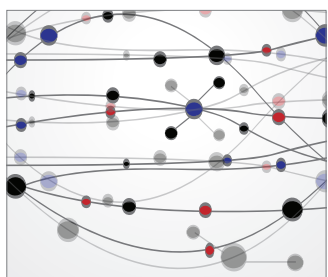

The Scientific World Journal
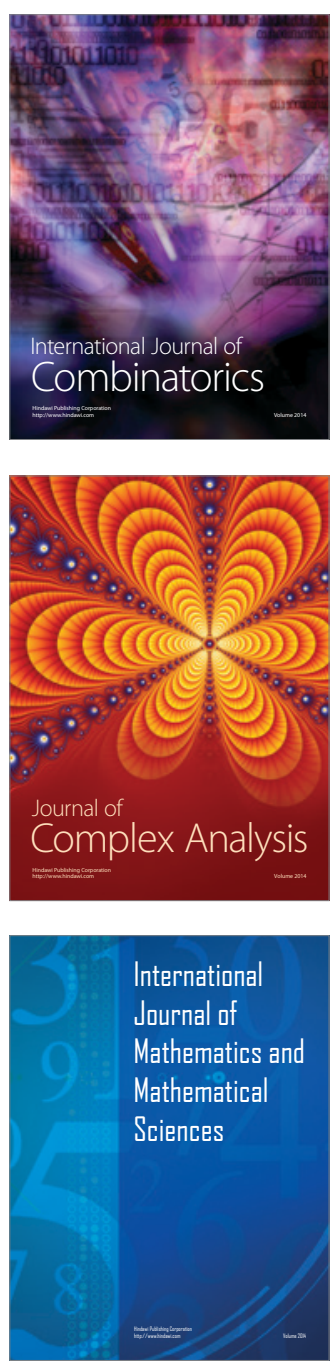
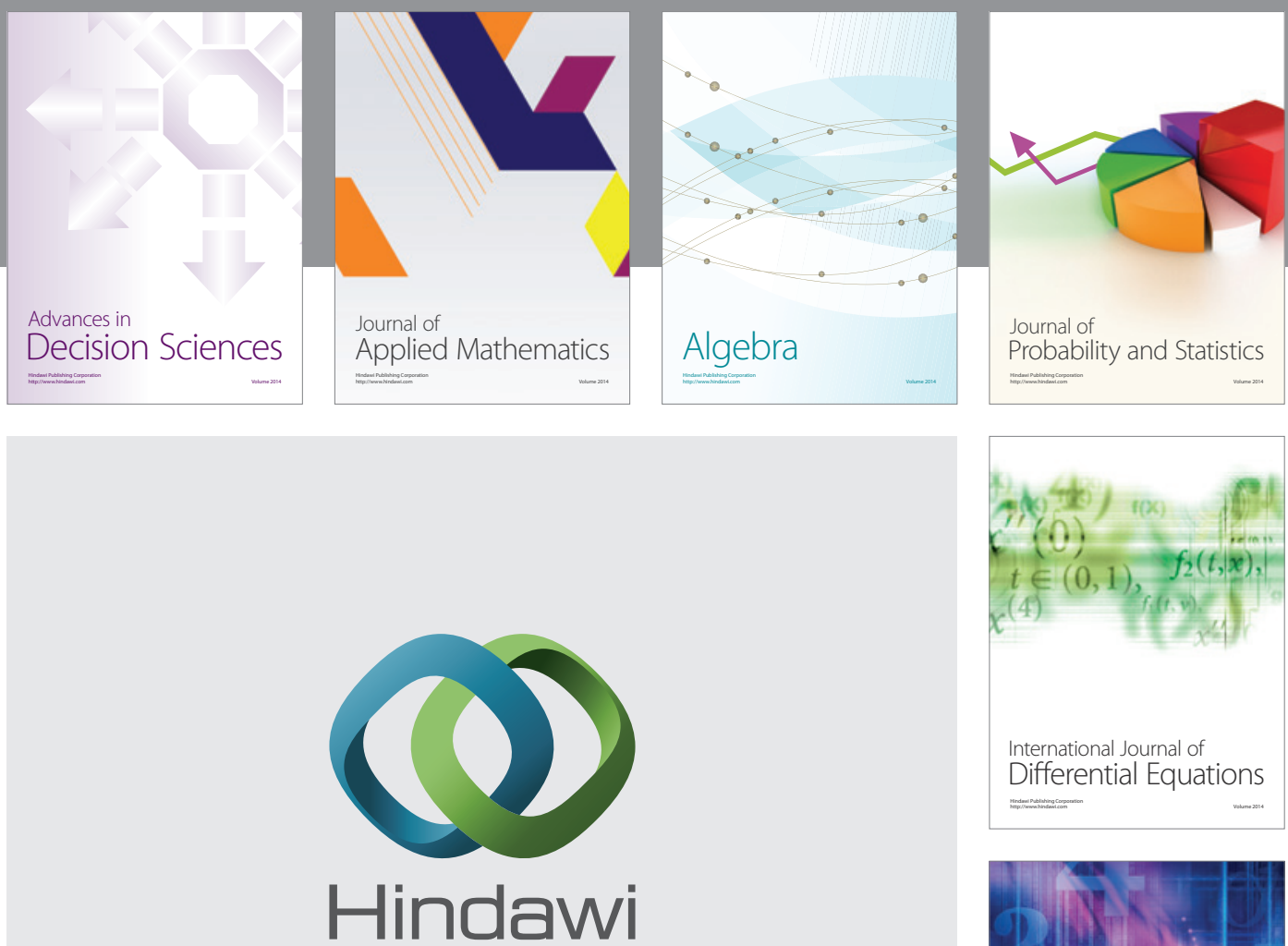

Submit your manuscripts at http://www.hindawi.com
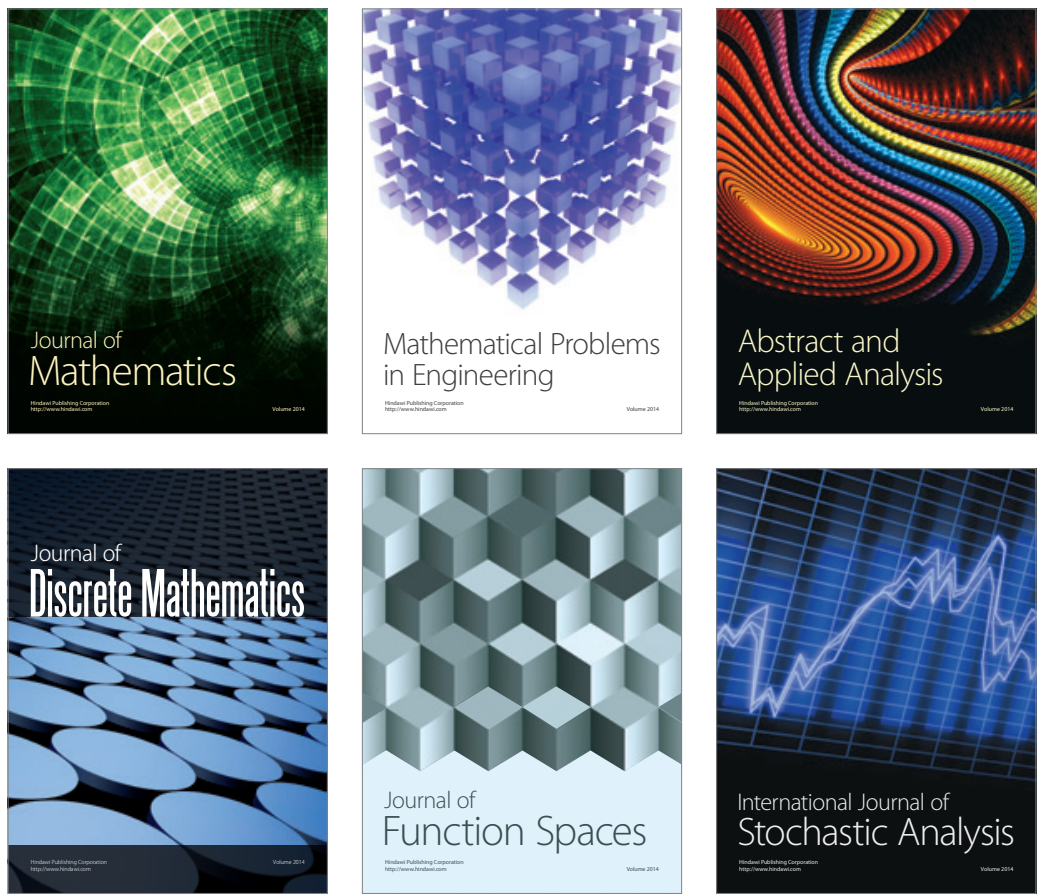

Journal of

Function Spaces

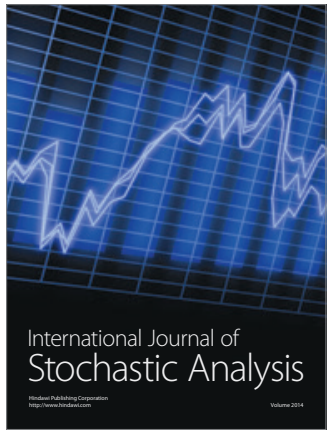

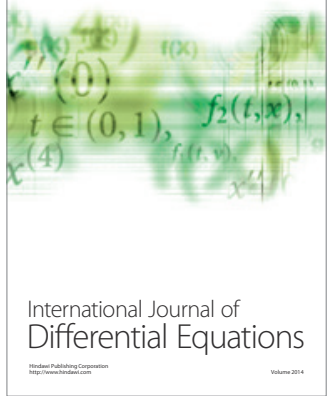
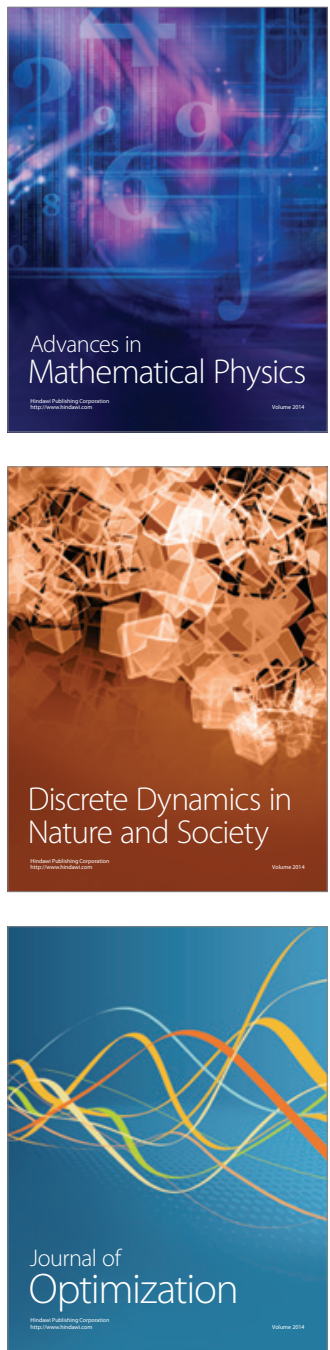